This is an electronic reprint of the original article. This reprint may differ from the original in pagination and typographic detail.

Author(s): Rice, Ronald E.; Evans, Sandra K.; Pearce, Katy E.; Sivunen, Anu; Vitak, Jessica; Treem, Jeffrey $W$.

Title: $\quad$ Organizational Media Affordances : Operationalization and Associations with Media Use

Year: $\quad 2017$

Version:

Please cite the original version:

Rice, R. E., Evans, S. K., Pearce, K. E., Sivunen, A., Vitak, J., \& Treem, J. W. (2017).

Organizational Media Affordances : Operationalization and Associations with Media Use. Journal of Communication, 67(1), 106-130. https://doi.org/10.1111/jcom.12273

All material supplied via JYX is protected by copyright and other intellectual property rights, and duplication or sale of all or part of any of the repository collections is not permitted, except that material may be duplicated by you for your research use or educational purposes in electronic or print form. You must obtain permission for any other use. Electronic or print copies may not be offered, whether for sale or otherwise to anyone who is not an authorised user. 


\title{
Organizational Media Affordances: Operationalization and Associations with Media Use
}

\author{
Ronald E. Rice, $\mathrm{PhD}$ \\ Dept. of Communication, University of California \\ Santa Barbara, CA 93106-4020 \\ rrice@,comm.ucsb.edu \\ Sandra K Evans, $\mathrm{PhD}$ \\ Department of Communication, Cal Poly Pomona \\ Pomona, CA 91768 \\ skevans@,cpp.edu \\ Katy E. Pearce, $\mathrm{PhD}$ \\ Department of Communication, University of Washington, Box 353740 \\ Seattle, WA 98195-3740 \\ kepearce@uw.edu \\ Anu Sivunen, $\mathrm{PhD}$ \\ Department of Communication (L), P.O. Box 35 \\ 40014 University of Jyväskylä, Finland \\ anu.e.sivunen@jyu.fi \\ Jessica Vitak, PhD \\ College of Information Studies, University of Maryland \\ College Park, MD 20742 \\ jvitak@umd.edu \\ Jeffrey W. Treem, PhD \\ Department of Communication Studies, The University of Texas at Austin \\ 2504A Whitis Ave. (A1105) \\ Austin, TX 78712-0115 \\ jtreem@austin.utexas.edu
}

This is the author's accepted manuscript of the published article:

Rice, R. E., Evans, S. K., Pearce, K. E., Sivunen, A., Vitak, J., \& Treem, J. W. (2017). Organizational Media Affordances: Operationalization and Associations with Media Use. Journal of Communication, 67(1), 106-130. 
Running head: ORGANIZATIONAL MEDIA AFFORDANCES

\title{
Organizational Media Affordances:
}

\section{Operationalization and Associations with Media Use}

\begin{abstract}
The concept of affordances has been increasingly applied to the study of ICTs in organizational contexts. However, almost no research operationalizes affordances, limiting comparisons and programmatic research. This paper briefly reviews conceptualizations and possibilities of affordances in general and for media, then introduces the concept of organizational media affordances as organizational resources. Analysis of survey data from a large Nordic media organization identified six reliable and valid organizational media affordances: pervasiveness, editability, self-presentation, searchability, visibility, and awareness. Eight media scales based on frequency of use of 10 media within each of three organization levels were differentially associated with these affordances. The conceptualization, measurement approach, and results from this study provide the foundation for considerable future organizational communication and ICT research.
\end{abstract}

keywords: affordances, organizations, ICTs, media use, operationalization 


\section{Organizational Media Affordances:}

\section{Operationalization and Associations with Media Use}

With the introduction of computers and digital transmission networks, new forms of organizational and personal information and communication technologies (ICTs) have arisen, diffused, and been incorporated into organizations' and people's lives. These include computer conferencing systems, online bulletin boards, email, group support systems, voice mail, intranets, videoconferencing, virtual collaboration, texting, mobile phones, and social media. Recently, scholars have considered how the affordances of ICTs can provide a useful framework to understand organizational media use and implications. In these studies, researchers evaluate the relationship between users and technology to understand the various ways ICTs are adopted, appropriated, and reinvented by users, as well as how specific affordances of ICTs are associated with social and communicative outcomes. These studies may be especially beneficial to organizational communication research because they provide new insights into how technology use shapes members' work processes and interactions, and how technology use is shaped by members' perceptions and needs.

The present study extends communication scholarship by adopting an affordances perspective, and contributes to theory, by explicating the conceptual foundations of organizational media affordances, considering them as organizational resources rather than assessing each affordance in a 1-to-1 relation to a specific medium, developing operationalizations of a central set of affordances useful for surveys and interviews, and assessing how use of various organizational media at three organizational levelsinterpersonal, group, and organizational — is associated with those affordances. 


\section{Problem Statement, Review and Research Questions}

\section{The Nature of Media}

Major theoretical approaches to understanding the nature of media include media ecology (McLuhan, 1964), educational media typologies (Bretz, 1971), media symbol systems and cognitive processing (Salomon, 1979), social presence (Short, Williams \& Christie, 1976; Rice, 1993), media richness (Daft \& Lengel, 1986), media attributes (Rice, 1987), task-technology attributes (Nass \& Mason, 1990), social construction of technology (Bijker, Hughes, Pinch \& Douglas, 2012; Fulk, 1993), adaptive structuration (DeSanctis \& Poole, 1994), and uses and gratifications (Flanagin \& Metzger, 2001; Sundar \& Limperos, 2013). Each of these approaches has extended our understanding of how and why individuals use particular media and how and why different media may be associated with particular outcomes, recognizing the ways media use may be differentially shaped by material aspects of technologies (i.e., features), and users' perceptions and motivations. However, even among theoretical approaches that aim to balance the role of technologies' features and users' perceptions and uses, scholars often privilege one side of the technology-user relationship () or conflate the two (as Faraj \& Azad, 2012, and Leonardi \& Barley, 2008, note). A focus on media affordances offers a theoretical grounding in the relationships between users and technology, and therefore a middle path between deterministic and constructivist stances. Yet the conceptualization of affordances is inconsistent (Author1, year), and operationalization of affordances is rare, making it difficult to compare studies and understand results.

Thus this study provides an initial attempt to operationalize media affordances in organizational contexts. Our construct measurement and validation process follows 
Mackenzie, Podsakoff, and Podsakoff's (2001) 10 recommendations including: 1)

develop the conceptualization, 2) generate items to represent the construct, 3) assess the content validity of the items, 4) specify the measurement model, 5) collect and analyze data, 6) purify and refine scales, and 8) reassess scale validity (groups comparisons and predictive associations). For this case study we did not use steps 7 and 9 , which require obtaining additional samples, nor step 10, which proposes obtaining norms for the scales for relevant populations, deemed inappropriate for the concept of affordances.

\section{Conceptualizing Affordances (step \#1)}

The concept of affordances was first defined by Gibson (1979) in the context of the natural environment. Gibson intended affordance to mean an "action possibility available in the environment" (McGrenere \& Ho, 2000, p. 1). For Gibson, affordances exist as an action possibility independent of an actor's perception and experiences; do not change when an actor's needs and goals change, but they are relative to each actor's perceptions and capabilities for action; exist or do not, without distinctions of degree or extent; and can be nested (comprising other action possibilities). Later, Norman (1988) developed a human-centered design perspective on affordances. Affordances are perceived, not actual; vary in degree or extent; and may be shaped by users through applying functional affordances that may be nested within more general affordances (McGrenere \& Ho, 2000).

Excellent reviews, explications, and comparisons of the affordance concept indicate varying and even opposing uses of the term (Bonderup-Dohn, 2009; Burlamaqui \& Dong, 2015; Chemero, 2011; Faraj \& Azad, 2012; McGrenere \& Ho, 2000; Oliver, 2005; Rietveld \& Kiverstein, 2014). Debates include whether affordances exist 
independently of the actor's perceptions (or capability for becoming aware of the affordance) and effectivities (dispositions for actualizing the affordance); whether the core relation is between actor properties and environment properties, or between actor properties and situation aspects; and whether affordances are properties of the object/environment, a latent capability emerging in a particular context, or specific to the actor/species. This ambiguity has, however, also allowed for the evolution of the concept in a range of disciplines (Author1, year; Author2, year).

For many researchers, a particular role of an affordance depends on whether and how the agent perceives the affordance, and thus how the agent applies it. Thus, we need measures of those perceptions (Wang, Carte \& Schwarzkopf, 2015). For example, Gaver (1991; see also McGrenere \& Ho, 2000) distinguished between information in the affordance itself (usefulness), and mediating information about the affordances (usability, such as labels, implementer or other user suggestions, the context), giving rise to four kinds of affordances: correct rejections, perceptible, hidden, and false affordances. Further, the same object may offer different affordances to different contexts and actor groups (Faraj \& Azad, 2012; Oliver, 2005; Oostervink, Agterberg, \& Huysman, 2016). An affordance can have both positive and negative, intended and unintended, and shortterm and long-term connotations; it may both enable and constrain action (Conole \& Dyke, 2004; Majchrzak, Faraj, Kane, \& Azad, 2013; Oostervink, Agterberg, \& Huysman, 2016). Affordances may be nested, temporally or spatially interdependent, and bundled into sets of interrelating affordances and outcomes (Strong et al., 2014).

Emphasizing a design perspective on affordances, Burlamaqui and Dong's review (2015) generates five common foundational elements: artefact, agent, environment, 
perception, and potential use. Pozzi, Pigni and Vitari (2014) distinguish and summarize four main aspects of affordances: their existence, perception, actualization, and effect. Other researchers are now attending to the importance of actualization of affordances (Strong et al. 2014; Volkoff \& Strong, 2013), which may depend on a wide variety of conditions, agents, and goals.

Media affordances. Affordances inherently involve communication. From Norman's perspective, a designer attempts to communicate to the user about capabilities of the artefact through affordances, both inherent in the artefact, and through information in or on the artefact about the affordances (Burlamaqui \& Dong, 2015; Gaver, 1991). Of course, media afford co-construction and sharing of intersubjective meaning (Suthers, 2006), such as between organizational members. Organizational researchers have more recently begun applying the concept of affordances to explain uses, context, and implications of organizational media (i.e., Goh, Gao \& Agarwal, 2011; Leonardi, 2013; Majchrzak et al., 2013; Pozi, Pigni, \& Vitari, 2014; Treem \& Leonardi, 2012; Volkoff \& Strong, 2013). Results describe a wide range of possible and overlapping affordances; in just the case of mobile phones, over 50 (see, for example, Author3, year, Table 1). Typically, affordances are measured in relation to a specific ICT, at the individual level (Ellison, Gibbs, \& Weber, 2015) and, with some exceptions, at a single point in time (Ellison \& boyd, 2013; Ellison \& Vitak, 2015). Others propose general affordances, unrelated to specific media. For example, Sundar's (2008) MAIN model proposes four broad media affordances: modality, agency, interactivity and navigability, which cue cognitive heuristics about credibility assessments. Thus media affordances are relationships among action possibilities to which agents perceive they could apply a 
medium, within its potential features/capabilities/constraints, relative to the agent's needs or purposes, within a given context.

Organizational media affordances. Some have suggested higher-order categories of affordances. Leonardi (2013) proposed individualized (one person's engagement of an affordance), collective (enacted by a group, whether pooled or interdependent), and shared (a group perceives and appropriates a new technology's features in a similar way) affordances. Bardner (2001) proposed the concept of social affordances, whereby a group's social aspects and norms interact with an object's properties to facilitate specific kinds of group relations. At an organizational level, Zammuto, Griffith, Majchrzak, Dougherty, and Faraj (2007) introduced affordances for organizing to describe how the relationship or "intertwining" between IT and organizational systems impacted organizations, an argument that echoes Orlikowski and Scott's (2008) claim that the social and material are "constitutively entangled" (p. 752).

We propose the concept of organizational media affordances: relationships among action possibilities to which agents perceive they could apply a medium, within its potential features/capabilities/constraints, relative to the agents' needs or purposes, aggregated within or across media contexts, and within or across organizational contexts. This concept makes three central assumptions. First, agents perceive the extent to which a salient affordance is available within organizational contexts to accomplish their work through use of different available media. Thus, organizational media affordances are organizational resources. Second, rather than a 1-to-1 linkage between a single technology (platform or medium) and a single affordance, organizational media affordances can be associated with a single, multiple, or groups of organizational media. 
Organizational members increasingly use multiple communication media during the workday, sometimes simultaneously or sequentially, and choose different media depending upon both communication goals and relational partners (Leonardi, Neeley, \& Gerber, 2012; Stephens, Sørnes, Rice, \& Browning, 2008). Third, relations between affordances and media use occur within at least three organizational communication contexts (Rice \& Leonardi, 2013): interpersonal, group, and organizational levels. Some media use — and thus affordances — vary across these levels due to contextual needs for collaboration and interdependence, physical and temporal proximity, number of interaction partners, commonness of activities, and formal reporting relationships, while other use may be relevant to multiple levels throughout the organization.

Interpersonal level. Organizational members frequently engage in dyadic or small group communication (e.g., supervisor-subordinate). Mediated communication with a supervisor may overcome constraints of time, location, and knowledge, enabling more resources (Kubicek, Korunka, Paškvan, Prem, \& Gerdenitsch, 2014).

Group level. The group level can relate to work groups, teams, or departments. For example, Bradner, Kellogg, and Erickson (1999) define affordances as "the relationship between the properties of an object and the social characteristics of a group that enable particular kinds of interaction among members of that group" (p. 154), moving beyond the common dyadic actor-object relationship at the interpersonal level.

Organizational level. Gibbs, Rozaidi, and Eisenberg (2013) explored affordances at the organizational level by assessing dialectical tensions emerging from interview data about social media use in a technology organization. Treem and Leonardi (2012) explicated the role of four affordances - visibility, persistence, editability, and 
association - as potentially significant influences on central organizational communication processes, such as socialization, knowledge sharing, and power relations.

\section{Operationalizing Organizational Media Affordances}

The value in operationalizing organizational media affordances lies in the potential for measuring and analyzing them across multiple contexts and including affordances in multivariate models of ICT adoption, use, and outcomes. Despite the growing analysis of ICT affordances in organizational contexts noted above, little research has attempted to measure a consistent and broad set of affordances. Respondents typically do not themselves identify affordances, instead reporting their motivations for, purposes of, or particular uses of a medium, to which researchers then apply affordance labels. An analysis of 120 articles at least mentioning media affordances (table available from the authors) identified only three studies quantitatively measuring affordances (Kuo, Tseng, Tseng, \& Lin, 2013; McEwan \& Fox, 2015; Wang et al., 2015), and these involved a small number of affordances, media, and/or contexts.

\section{Research Questions}

Thus we ask: RQ1: How might we measure organizational media affordances? RQ2: What reliable, valid, and primary organizational media affordances emerge from those measures? RQW3: How do organizational media affordances associate with use of different or separate sets of media in different organizational contexts?

\section{Method}

Measures (steps \#2 and \#3)

Organizational media affordances. Consistent with our goal of developing a better understanding of affordances as a construct, our process of identifying and creating 
appropriate items reflecting organizational media affordances was initially grounded in the espoused findings of extant literature. We were then guided by an iterative, and abductive logic in which we developed constructs from these findings, and reflexively considered, reconsidered, and adjusted labels to test our assumptions and arrive at a plausible and appropriate representation of the material (Charmaz, 2006). Thus this approach is a mix of a priori and emergent coding. The concept of affordance is an existing coding domain, but the specific affordances and their groupings are emergent.

Before discussions with the organizational contact, to identify and create appropriate items reflecting organizational media affordances, we identified 79 terms in prior literature that referred to, or were named as, a media/ICT affordance. Based on their use, we converted those terms into phrases (e.g., "find out about new information through links with information you do know"). Using iterative discussion among three project researchers, we grouped these phrases into 13 tentatively labeled common affordancesassociation, awareness, content mode, editability, multitasking, persistence, personalization, pervasiveness, scalability, searchability, sharing, value, visibility. The survey did not include these tentative affordance categories; they were used only to group and distinguish the phrases for comparison. Reviewing the items and categories, we decided that several were not affordances (content mode, multitasking), were not widely relevant or were represented in another category (scalability), or were better conceptualized as an aspect of another affordance (sharing). We also added a new category (signaling) to distinguish several items from visibility and reworded some items for consistency and clarity. For the survey, we sought maximum variation in a small number of meaningful and clear items, consistent with the qualitative design approach of 
maximizing theoretical variance (Charmaz, 2006), given limits on the survey length. Therefore, each researcher, within each category, ranked the item most representative of the category and the two items most different from that one and from each other. We reviewed these rankings and reached consensus on two to five items for each category, resulting in a final set of 31 items. Items were reworded into a consistent format, beginning with the opportunity for action and emphasizing the essential affordance (e.g., "be aware of activities, opinions, or locations of others") (see Table 1). We then added a 7-point response scale $(1=$ strongly disagree to $7=$ strongly agree) to reflect the idea that perceptions of affordances reflect degree or extent, rather than simple existence or nonexistence.

--- Table 1 goes about here ---

Media use. Based on prior studies and discussions with the organizational contact to insure we included available and relevant media, we developed measures indicating frequency ( $1=$ never to $9=$ many times a day) of using 10 media available within the organization, each within three increasingly narrow contexts: employees outside of department but within the organization, employees within one's department, and interpersonally with one's supervisor. Media included face-to-face one-on-one, face-toface meetings, send/receiving email, telephone calls, short messages (including text messages, Google Hangout chat, other chat programs), conference calls without video, conference calls with video, the organization's intranet, WhatsApp, and external social media for work-related matters.

Data (step \#5) 
Case site. We conducted the study in a Nordic public sector broadcasting company (NPB) employing more than 3300 people. NPB is distributed across 25 locations nationally. It operates several television and radio channels and produces news and current affair programs, documentaries, and educational and children's programming.

Collection. The survey was sent from one researcher's email address as a web link to all NPB employees. The message included an invitation letter to participate in the study, contact information for NPB's contact person, and a link to NPB's intranet site for a detailed description of the study. The survey was open for two weeks. We also sent two reminders via email to all participants who had not yet responded to the study.

Sample. We received 461 surveys (450 usable) out of 3394 invitations (response rate: $13.6 \%)$. Of those, over half (54.2\%) were female; $23.5 \%$ had upper secondary or vocational education, $27.1 \%$ university-applied sciences, and $49.6 \%$ university; the mean age was 49.1 ( $\mathrm{SD}=9.2)$; 17.8\% were supervisors; they worked in six organizational units, 24 professions, and 37 departments; and they worked away from the office $18.1 \%$ of the time $(\mathrm{SD}=20.3)$. Thus they are not statistically representative, but do provide good diversity across the organization.

Results (steps \#4, \#5, \#6, \#8)

\section{Organizational Media Affordances}

Table 2 provides the item descriptives, principal components (EFA) results, mean scale descriptives and Cronbach reliabilities of the affordances measures. EFA criteria included eigenvalues over 1.0, loadings at least .60 (or close if there is conceptual grounding for including the item) on one component and less than .40 on any other component. 


\section{--- Table 2 goes about here ---}

Six affordances emerged, ranked by decreasing mean agreement: pervasiveness, editability, self-presentation, searchability, visibility, and awareness. Though the items for editability and self-presentation loaded on one component, we distinguished the two concepts (supported by the CFA, noted below), based upon the first three and the last four items, respectively. The respondents perceived distinct sets of affordances, they agreed that these are all possible actions, and they perceived some as more possible than others. We can therefore view these as organizational resources. A second-order principal components analysis of the six scales indicated one underlying factor, explaining $58.0 \%$ of the variance (eigenvalue $=3.48$ ), with a resulting overall Cronbach's $\alpha$ of 0.85 .

To verify the EFA results, we conducted a measurement model (using AMOS 22), with each of the items included as reflective indicators of their respective six unobserved constructs, and the six constructs indicating one underlying common construct. All item loadings in Model 1 were significant $(p<.001)$, and the overall model fit was marginally acceptable $\left(X^{2}(d f 269)=1121.3, p<.001 ; \mathrm{CMIN} / \mathrm{df}=4.27, \mathrm{TLI}=.88, \mathrm{CFI}=.90, \mathrm{RMSEA}\right.$ $=.08, \mathrm{AIC}=1283.3)$. Model 2 removed four low-loading items while retaining at least three items for each affordance. Model 2 provided an acceptable fit $\left(X^{2}(d f 183)=578.4\right.$, $p<.001 ; \mathrm{CMIN} / \mathrm{df}=3.16, \mathrm{TLI}=.93, \mathrm{CFI}=.94, \mathrm{RMSEA}=.07, \mathrm{AIC}=716.4)$, and was significantly better than Model $1\left(X^{2}(d f 86)\right.$ difference $\left.=542.9, p<.001\right)$.

To evaluate whether there is one underlying affordance concept as indicated from the exploratory second-order factor analysis and Model 2, Model 3 used only the six firstorder affordances. This model was a good fit $\left(X^{2}(d f 174)=513.8, p<.001 ; \mathrm{CMIN} / \mathrm{df}=\right.$ $2.95, \mathrm{TLI}=.93, \mathrm{CFI}=.95, \mathrm{RMSEA}=.065, \mathrm{AIC}=669.84)$, and significantly better than 
Model $2\left(X^{2}(d f 9)\right.$ difference $\left.=64.6, p<.001\right)$. Thus the six affordances are conceptually and empirically distinct, but interrelated, concepts. Figure 1 shows this final model, with the standardized regression weights; all entries are significant at $p<.001$. All affordance means are significantly $(p<.001)$ higher than the middle response choice of 4.0.

--- Figure 1 and Table 3 go about here ---

All factor loadings in the CFA measurement model exceed the recommended minimal value of 0.7 . Table 3 shows that Cronbach's alpha and composite reliabilities range from 0.82 to 0.96 , the average variance extracted (AVE) ranges from .63 to .77, and the square roots of the construct AVEs are all greater than the cross-correlations. These results provide evidence of scale reliability and convergent and discriminant validity.

\section{Organizational Media Use}

Table 4 provides the descriptives, EFA results, mean scale descriptives, and reliabilities for the media use items. Several resulting components reflect media use within separate organizational levels, while others reflect media use across levels. Each of the three levels had its own basic set of media: face-to-face, meeting, email, and phone. For department-level items, though the first two and second two media loaded on separate components, we combined them for comparability to the other levels, resulting in a higher alpha than for either pair of items. Five less traditional sets of media used across all three levels emerged: conferencing, external social media, WhatsApp, intranet, and texting. Communication was frequent across the three levels of basic media $(M=6.22,7.04$, and 5.34), less frequent for texting $(M=4.85)$, and low for external social media (3.23), conferencing without video (2.25), conferencing with video (2.25), 
organizational intranet (2.07), and WhatsApp (1.77). Thus perceptions of affordances associated with these last media may not be based on much experience.

--- Table 4 goes about here ---

We could not find a confirmatory factor analysis measurement model to fit the media use items, both with and without a second order common factor. Thus we consider each media scale as an index of the respective media use, identified through EFA.

Additional Tests (\#5 \& \#8)

Common method bias. Using the minimal tests of Harman's single factor test and multicollinearity (Bagozzi et al., 1991; Podsakoff, MacKenzie, Lee, \& Podsakoff, 2003), we found no evidence of common method bias.

Known-groups comparisons. We conducted independent samples t-tests of mean differences for the six affordances scales for two groupings: supervisory position ( $82.2 \%$ no, $17.8 \%$ yes), and percent of time working away from the office (dichotomized into $50 \%$ did so $<=14 \%$ of the time, $50 \%$ did so $>14 \%$ ). There is no strong theoretical reason why affordances should vary by supervisory role, but we might expect differences between low and high levels of working away from the office because of the relationships between varying media affordances and user needs related to communicating and accomplishing work when away from the office. Indeed, there were no significant differences between supervisors and non-supervisors. There were, however, two significant differences by working away from the office, with those doing so more agreeing more about the possibility of the self-presentation affordance $(t(399)=-2.2, p<$ $.05)$ and of the pervasiveness affordance $(t(407)=-2.6, p<.05)$. So the affordances seem 
fairly robust across two main organizational roles, but with some reasonable variations across work contexts.

Predictive associations. Table 5 presents correlations between the media affordances and media use scales. Because of the lack of CFA model fit for the media use measures, we cannot use an overall SEM model; therefore, we used the mean scales of each of the CFA construct affordance items and EFA component media use items. Over half (26) were significant, all positive.

--- Table 5 goes about here ---

Affordances. All affordances are positively associated with texting, and all are positively associated with at least three media. Specific affordances exhibit different relational patterns. Visibility is afforded through conferencing, external social media, the intranet, and texting. Editability is afforded through conferencing, the basic set of media at the organizational and departmental level, and texting. Self-presentation correlates with conferencing, external social media, and texting. Awareness is similarly associated with conferencing, external social media, and texting, but also basic media at the supervisor level, and the intranet. Pervasiveness is afforded through conferencing, basic media at the organizational and supervisor levels, external social media, texting, and WhatsApp. Finally, searchability is correlated with external social media, the intranet, and texting.

Media use. As noted above, it is difficult to know how the low use of external social media, conferencing, the intranet, and WhatsApp reflect perceptions of their affordances. The correlations, however, show consistent relationships between texting and all affordances, conferencing with all but searchability, and external social media with all but editability. The intranet and WhatsApp were less associated with affordances. 
The basic set of media scale was differentially associated with affordances at each organizational context. The organization-wide basic media scale was significantly correlated with editability, awareness, and pervasiveness; within-department basic media with editability and pervasiveness; and basic media use with one's supervisor with awareness.

\section{Discussion}

\section{Contributions, Implications, and Future Research}

This study makes several contributions to affordances research in organizational settings. The survey instrument provides a large set of items for assessing organizational media affordances. Survey items elicited respondents' perceived affordances in a quantitative manner, and in a way that was not tied to a single medium. We assessed different levels and types of media use, from basic communication within three levels to social media organization-wide. The sample involved experienced ICT users across a broad array of professions and departments in a major media organization.

Concerning RQ1, these findings indicate that possible actions with media can be distinctly, reliably, and validly associated with a primary set of affordance types within an organizational context. These results also provide support for the notion of affordances as organizational resources. Empirically deriving reliable measures of affordances within an organizational context allows researchers to ask when or how affordances vary across different organizational media and organizational contexts while retaining ecological validity that they are representing the views of organizational members. As Walther (2012, p. 191) commented, affordances "can only [help develop theory] if they provide a stable base for comparisons." Analyzing the affordance perceptions of individual media 
users, as opposed to inferring affordances from observed or presumed behaviors, is consistent with both Gibson's and Norman's conceptualizations of affordances as perceived by actors confronting objects in an environment.

RQ2 asked about types of organizational media affordances. From a large pool of items based on prior literature, our investigation identified six organizational media affordances: visibility, editability, self-presentation, awareness, pervasiveness, and searchability. However, there were at least four media affordance categories referenced in previous research that did not emerge as unique affordances from responses by the workers at NPB: association, evaluatability, sharing, and signaling. Also, based on the CFA analyses, the resulting scales did not include some affordance survey items. The two association items nearly equally load on both awareness and pervasiveness. One persistence item (find information about prior NPB projects) nearly loaded high enough to be included with the awareness scale or the searchability scale. The three sharing items loaded about equally on the visibility, and editability/self-presentation scales. All of these items could be included in the respective scale of their highest loading with no decrease and in some cases a small increase in the respective scale reliability. Because of their minimal loadings and cross-loadings, we did not include these.

These and other affordances might emerge from a different, more distinct, and larger set of items (e.g., we did not include items for the initial affordances of scalability and multitasking). Future research could consider more distinct items representing these affordances, either allowing those affordances to emerge as separate measures, or showing that while identified in qualitative studies of a few affordances, may not be as conceptually or empirically distinct as presumed. Because human capabilities are broad, 
diverse, and changing, affordances will improve and increase, in turn affecting the environment, generating new affordances and action possibilities (Rieteveld \& Kiverstein, 2014). Indeed, "every artefact has an uncountable number of affordances" (Burlamaqui \& Dong, 2015, p. 306); thus, there can be no final, exhaustive set of affordances (Oliver, 2005). We also note that affordance perceptions may become so routinized and instinctual (Burlamaqui \& Dong, 2015) that they become "invisible" (Ortmann \& Kuhn, 2010). Thus no measure (perceptual, observed, reported, or inferred) can capture all potential affordances.

Future research should obtain additional samples to re-assess and cross-validate the media use and affordance constructs (\#7, \#8, \#9 in Mackenzie, Podsakoff, and Podsakoff's (2011) process). However, given the large number of items included, it may be difficult to replicate these within other studies, especially if they include a variety of other measures.

The initial tentative 11 affordances correspond with much of the literature. The emergence of the six affordances from respondents' perceptions, and their overlap with at least four others in the literature, show good correspondence between the literature and our data. Further, the results suggest affordances can be shared by individuals in an organization - workers largely agree on what actions technologies can, and cannot, support (Leonardi, 2013). They also suggest that, in addition to the traditional research approach of assessing affordances of each specific communication channel, affordances can also be conceptualized as organizational resources, fostered by an array of available communication channels, with multiple media associated with the same affordance, and multiple affordances associated with the same medium. 
By operationalizing affordances in this manner, and proposing reliable and valid measures for them, scholars can develop mid-range theories regarding the affordances of organizational media across organizational contexts, allowing future work to test the scope and applicability of affordances as media and the environments of users change. Showing that affordances are not limited to specific technologies could make empirical research even more valid and generalizable across organizational contexts and changing media platforms and technologies (Ellison \& Vitak, 2015).

Further, the boundaries of affordances are permeable, as indicated by their intercorrelation, reinforcing the view that affordances of media may occur in conjunction with, or even facilitate or constrain, other affordances (Schrock, 2015; Treem \& Leonardi, 2012). Future work should continue to explore additional affordances, their inter-relationships, and their relationships with media. A comprehensive organizational media affordances approach should analyze media affordances over time, since the relationships between people, media, context, and purposes are inherently dynamic.

Regarding RQ3, affordances are differentially associated with a variety of media used across organizational levels, and differentially associated with a set of basic media within supervisory, departmental, and organizational levels. One implication is that just as affordances can be considered an interrelated set of organizational resources, so too can media. Rather than considering each medium as providing distinct uses and affordances, similarly used media may provide related affordances, depending on the context, user, and purpose. One way to interpret this is that organizational media are flexible in how they support various actions. Rarely was a medium associated with only one affordance. This is consistent with Treem and Leonardi's (2012, Table 7.1, p. 149) 
view that an organizational medium vary from high to low in media affordances, and with Norman's view that affordances involve degree or extent. As affordances are conceptualized as action possibilities, non-significant correlations may reflect lack of actualization of some possibilities for some media. People may perceive an affordance, but choose or are constrained not to actualize it, for a variety of reasons, including the specific nature of the affordance in a given context (Strong et al., 2014).

Finally, future research should develop more comprehensive models of the shaping of affordance perceptions and actions (as has been done in much of the media theories noted earlier), and how affordances enable or constrain salient outcomes, such as knowledge sharing (Ellison et al., 2015; Leonardi, 2011; Majchrzak et al., 2013). For example, studies could incorporate organizational media affordance measures in one of the several more complete frameworks summarized above (Burlamaqui \& Dong, 2015; Pozzi, Pigni \& Vitari, 2014). Such analyses will help build new theories to predict and explain the relationship between people and organizational ICTs in the digital age.

\section{Limitations}

Although the sample is large and diverse, it consists of a small percentage of respondents from a single Nordic media organization. Thus, there are many sources of non-representation and contingency, such as generalizability to the organization, to types of work, and to organizational and national cultures.

Nearly all prior ICT affordance studies involve researchers qualitatively interpreting participants' observed or reported behaviors or discourse as a limited set of researcher-labeled affordances. Even in this study, though the affordance labels correspond to those in the literature, the researchers are labeling the six affordances, 
some of which include items initially associated with other tentative affordance labels, and some of which could be labeled differently. For example, "self-presentation" might also be labeled "identity management."

Some might reject, on epistemological grounds, that affordances should or can be assessed quantitatively and as distinct from specific technology-user-environment relations. For example, Bygstad, Munkvold, and Volkoff (2016) insist that affordances cannot be studied directly, but only through qualitative understanding of the associated events and issues. Some implication of their position is that it is very difficult to identify or predict all affordances, and it is not clear who should be defining or labeling the affordance, or even what the best label is (Stendal, Thapa, \& Lanamaki, 2016).

We suggest three justifications for our approach. First, relying on self-reports of perceived affordances is a common practice in studies of communication technology use. For instance, Gibbs et al. (2013) and Leonardi (2013) use interviews to evaluate and operationalize affordances in organizational settings. Using the survey measures to operationalize affordances, and to do so through perceptions, shares this focus on individuals' perceptions of technologies. As Norman and other researchers reject the assumption that affordances either exist or do not exist (Gibson's position), it is appropriate to consider the extent to which agents perceive the degree or extent of affordance possibilities.

Second, scholars have noted the need to explore different ways of measuring affordances, including the potential for developing quantitative methods (Wang et al., 2015). By creating a uniform way of gaining knowledge into the perceived possibilities 
of action across a large group of employees and media, we can develop more reliable representations of the breadth of affordances in an environment.

Third, taking a quantitative approach addresses some limitations of qualitative methods, such as the difficulty of 1) gathering qualitative data about a large number of individuals, 2) comparing within or across organizations, 3) working toward generalizations and facilitating hypothesis testing, and 4) replication and comparisons with other studies. Nonetheless, without engaging paradigm issues or mixed-methods design perspectives, certainly quantitative and qualitative approaches to understanding affordances complement each other. Indeed, future research may complement this framework with more in-depth and qualitative understanding of each of the affordances and their relation to various media, as Leonardi (2014) has done with visibility.

\section{Conclusion}

With the increasing pervasiveness of organizational ICTs across all aspects of our lives, it is critical to empirically evaluate users' relationships with these technologies in ways that account for the attributes and affordances in mediated spaces. Within organizations, we have seen dramatic shifts over the last few decades in communication, collaboration, and information processes, thanks in part to new technologies bridging geospatial and temporal boundaries. This study highlights the powerful role of an affordance framework for analyses of media use in organizations. We extend existing research in this space with the creation and evaluation of valid and reliable organizational media affordance scales, and show how these affordances differentially associate with 10 types of media use across and within three organizational levels. 


\section{References}

Author1 (year).

Author2 (year).

Author3 (year).

Bagozzi, R.P., Yi, Y., \& Phillips, L.W. (1991). Assessing construct validity in organizational research. Administrative Science Quarterly, 36, 421-458.

Bijker, W. E., Hughes, T. P., Pinch, T., \& Douglas, D. G. (2012). The social construction of technological systems: New directions in the sociology and history of technology. Cambridge, MA: The MIT Press.

Bonderup-Dohn, N. (2009). Affordances revisited: Articulating a Merleau-Pontian view. International Journal of Computer-Supported Collaborative Learning, 4, 151170.

Boswell, W. R. \& Olson-Buchanan, J. B. (2007). The use of communication technologies after hours: The role of work attitudes and work-life conflict. Journal of Management, 33, 592-610.

Bradner, E., Kellogg, W. A., \& Erickson, T. (1999). The adoption and use of 'Babble’: A field study of chat in the workplace. In $E C S C W^{\prime} 99$ (pp. 139-158). Springer Netherlands.

Bretz, R. (1971). A taxonomy of communication media. Englewood Cliffs, N.J.: Educational Technology Publications.

Burlamaqui, L., \& Dong, A. (2015). The use and misuse of the concept of affordance. In J. S. Gero \& S. Hanna (Eds.), Design computing and cognition '14 (pp. 295-311). Switzerland: Springer International Publishing. 
Bygstad, B., Munkvold, B. E., \& Volkoff, O. (2016). Identifying generative mechanisms through affordances: A framework for critical realist data analysis. Journal of Information Technology, 31, 83-96.

Charmaz, K. (2006). Constructing grounded theory: A practical guide through qualitative analysis. London, UK: Sage.

Chemero, A. (2011). Affordances, etc. Chapter 7 in Radical embodied cognitive science. Cambridge, MA: The MIT Press.

Conole, G. \& Dyke, M. (2004). What are the affordances of information and communication technologies? ALT-J, Research in Learning Technology, 12, 113124.

Daft, R. L., \& Lengel, R. H. (1986). Organizational information requirements, media richness and structural design. Management Science, 32, 554-571. doi:10.1287/mnsc.32.5.554

DeSanctis, G., \& Poole, M. S. (1994). Capturing the complexity in advanced technology use: Adaptive structuration theory. Organization Science, 5, 121-147. doi:10.1287/orsc.5.2.121

Ellison, N. B., \& boyd, d. (2013). Sociality through social network sites. In W. H. Dutton (Ed.) The Oxford handbook of Internet studies (pp. 151-172). Oxford: Oxford University Press.

Ellison, N. B., Gibbs, J. L., \& Weber, M. W. (2015). The use of enterprise social network sites for knowledge sharing in distributed organizations: The role of organizational affordances. American Behavioral Scientist, 59, 103-123. doi:10.1177/0002764214540510 
Ellison, N. B., \& Vitak, J. (2015). Social network site affordances and their relationship to social capital processes. In S. S. Sunder (Ed.), The handbook of the psychology of communication technology (pp. 205-227). Maklen, MA: John Wiley \& Sons.

Ellison, N. B., Vitak, J., Gray, R., \& Lampe, C. (2014). Cultivating social resources on social network sites: Facebook relationship maintenance behaviors and their role in social capital processes. Journal of Computer-Mediated Communication, 19, 855-870. doi:10.1111/jcc4.12078

Faraj, S. \& Azad, B. (2012). The materiality of technology: An affordance perspective. In P. Leonardi, B. Nardi, \& J. Kallinikos (Eds.), Materiality and organizing: Social interaction in a technological world (pp. 237-258). Oxford, UK: Oxford University Press.

Flanagin, A., \& Metzger, M. (2001). Internet use in the contemporary media environment. Human Communication Research, 27, 153-181.

Fulk, J. (1993). Social construction of communication technology. Academy of Management Journal, 36, 921-950. doi:10.2307/256641

Gaver, W. W. (1991). Technology affordances. In Proceedings of the SIGCHI conference on Human factors in computing systems (pp. 79-84). New York: ACM. doi:10.1145/108844.108856

Gibbs, J. L., Rozaidi, N. A., \& Eisenberg, J. (2013). Overcoming the "ideology of openness": Probing the affordances of social media for organizational knowledge sharing. Journal of Computer-Mediated Communication, 19, 102-120. doi:10.1111/jcc4.12034 
Gibson, J. J. (1979). The ecological approach to visual perception. Hillsboro, NJ: Lawrence Erlbaum.

Goh, J. M., Gao. G. \& Agarwal, R. (2011). Evolving work routines: Adaptive routinization of information technology in healthcare. Information Systems Research, 22, 565-585.

Kubicek, B., Korunka, C., Paškvan, M., Prem, R., \& Gerdenitsch, C. (2014). Changing working conditions at the onset of the Twenty-First Century: Facts from international datasets. In C. Korunka \& P. Hoonakker (Eds.), The impact of ICT on quality of working life (pp. 25-41). Dordrecht, The Netherlands: Springer.

Kuo, F. Y., Tseng, C. Y., Tseng, F. C., \& Lin, C. S. (2013). A study of social information control affordances and gender difference in Facebook self-presentation. Cyberpsychology, Behavior, and Social Networking, 16, 635-644.

Leonardi, P. M. (2011). When flexible routines meet flexible technologies: Affordance, constraint, and the imbrication of human and material agencies. MIS Quarterly, $35,147-167$.

Leonardi, P. M. (2013). When does technology use enable network change in organizations? A comparative study of feature use and shared affordances. MIS Quarterly, 37, 749-775.

Leonardi, P. M. (2014). Social media, knowledge sharing, and innovation: Toward a theory of communication visibility. Information Systems Research, 25, 796-816. doi:10.1287/isre.2014.0536 
Leonardi, P. M., \& Barley, S. R. (2008). Materiality and change: Challenges to building better theory about technology and organizing. Information and Organization, 18, 159-176. doi:10.1016/j.infoandorg.2008.03.001

Leonardi, P. M., Neeley, T. B., \& Gerber, E. M. (2012). How managers use multiple media: Discrepant events, power, and timing in redundant communication. Organization Science, 23, 98-117. doi:10.1287/orsc.1110.0638

MacKenzie, S. B., Podsakoff, P. M., \& Podsakoff, N. O. (2011). Construct measurement and validation procedures in MIS and behavioral research: Integrating new and existing techniques. MIS Quarterly, 35, 293-334.

Majchrzak, A., Faraj, S., Kane, G. C., \& Azad, B. (2013). The contradictory influence of social media affordances on online communal knowledge sharing. Journal of Computer-Mediated Communication, 19, 38-55. doi:10.1111/jcc4.12030

McEwan, B. \& Fox, J. (2015, October). Why communication technologies matter: Developing a scale to assess the perceived social affordances of communication channels. Paper presented the National Communication Association conference, Las Vegas, NV.

McGrenere, J. \& Ho, W. (2000). Affordances: Clarifying an evolving concept. In Proceedings of GraphIcs Interface (pp. 1-8). Montreal.

McLuhan, M. (1964). Understanding media: The extensions of man. New York: Mentor. Nass, C., \& Mason, L. (1990). On the study of technology and task: A variable-based approach. In J. Fulk \& C. W. Steinfield (Eds.), Organizations and communication technology (pp. 46-67). Thousand Oaks, CA: Sage.

Norman, D. A. (1988). The design of everyday things. New York, NY: Doubleday. 
Oliver, M. (2005). The problem with affordance. E-Learning and Digital Media, 2, 402413.

Oostervink, N., Agterberg, M., \& Huysman, M. (2016). Knowledge sharing on enterprise social media: Practices to cope with institutional complexity. Journal of Computer-Mediated Communication, 21, 156-176.

Orlikowski, W. J., \& Scott, S. V. (2008). 10 sociomateriality: Challenging the separation of technology, work and organization. The Academy of Management Annals, 2, 433-474. doi:10.1080/19416520802211644

Ortmann, J., \& Kuhn, W. (2010). Affordances as qualities. Frontiers in Artificial Intelligence and Applications, 209, 117-130. doi:10.3233/978-1-60750-535-8117

Podsakoff, P. M., MacKenzie, S.B., Lee, J.-Y., \& Podsakoff, N. P. (2003). Common method biases in behavioral research: A critical review of the literature and recommended remedies. Journal of Applied Psychology, 88, 879-903. doi: $10.1037 / 0021-9010.88 .5 .879$

Pozzi, G., Pigni, F., \& Vitari, C. (2014). Affordance theory in the IS discipline: A review and synthesis of the literature. In Twentieth Americas Conference on Information Systems (12 pages), Association for Information Systems.

Rice, R. E. (1987). Computer-mediated communication and organizational innovation. Journal of Communication, 37, 65-94. doi:10.1111/j.1460-2466.1987.tb01009.x Rice, R. E. (1993). Media appropriateness: Using social presence theory to compare traditional and new organizational media. Human Communication Research, 19, $451-484$. 
Rice, R. E. \& Leonardi, P. M. (2013). Information and communication technology in organizations, 2000-2011. In L. Putnam, \& D. K. Mumby (Eds.), Sage handbook of organizational communication (pp. 425-448). Thousand Oaks, CA: Sage.

Rietveld, E., \& Kiverstein, J. (2014). A rich landscape of affordances. Ecological Psychology, 26, 325-352.

Salomon, G. (1979). Media and symbol systems as related to cognition and learning. Journal of Educational Psychology, 71, 131-148.

Schrock, A. R. (2015). Communicative affordances of mobile media: Portability, availability, locatability, and multimediality. International Journal of Communication, 9, 1229-1246.

Short, J., Williams, E., \& Christie, B. (1976). The social psychology of telecommunications. New York, NY: Wiley.

Stendal, K., Thapa, D., \& Lanamaki, A. (2016). Analyzing the concept of affordances in information systems. $46^{\text {th }}$ Hawaii International Conference on Systems Sciences (pp. 5270-5277). IEEE Computer Society.

Stephens, K., Sørnes, J. O., Rice, R. E., \& Browning, L. (2008). Discrete, sequential, and follow-up use of information and communication technology by advanced ICT users. Management Communication Quarterly, 22, 197-231.

Strong, D. M., et al. (2014). A theory of organization-EHR affordance actualization. Journal of the Association for Information Systems, 15, 53-85.

Sundar, S. S. (2008). The MAIN model: A heuristic approach to understanding technology effects on credibility. In M. J. Metzger \& A. J. Flanagin (Eds.), 
Digital media, youth, and credibility (pp. 73-100). Cambridge, MA: The MIT Press.

Sundar, S. S. \& Limperos, A. M. (2013) Uses and grats 2.0: New gratifications for new Media, Journal of Broadcasting \& Electronic Media, 57(4), 504-525.

Suthers, D. (2006). Technology affordances for intersubjective meaning making: A research agenda for CSCL. International Journal of Computer-Supported Collaborative Learning, 1, 315-337.

Treem, J. W. \& Leonardi, P. M. (2012). Social media use in organizations: Exploring the affordances of visibility, editability, persistence, and association. Communication Yearbook (vol. 36, pp. 143-189). New York, NY: Routledge.

Volkoff, O., \& Strong, D. M. (2013). Critical realism and affordances: Theorizing ITassociated organizational change processes. MIS Quarterly, 37, 819-834.

Walther, J. B. (2012). Commentary: Affordances, effects, and technology errors. In C. Salmon (Ed.), Communication yearbook (vol. 36, pp. 190-193). New York, NY: Routledge.

Wang, N., Carte, T. \& Schwarzkopf, A. (2015). How should technology affordances be measured? An initial comparison of two approaches. Twenty-first Americas Conference on Information Systems, Puerto Rico (14 pages). Association for Information Systems.

Zammuto, R. F., Griffith, T. L., Majchrzak, A., Dougherty, D. J., \& Faraj, S. (2007). Information technology and the changing fabric of organization. Organization Science, 18, 749-762. doi:10.1287/orsc.1070.0307 
Table 1

Final List of Survey Items, with Tentative Media Affordance Labels

Association: 1. use (web)links from information I know or am aware of, to find new information I did not know or wasn't aware of; 2. use (web)links from people I know or am aware of, to find new people I did not know or wasn't aware of Awareness: 3. be aware of the information others in my department have; 4 . be aware of the information others outside of my department have; 5 . be aware of activities, opinions, or locations of others; 6 . keep up-to-date with the progress of projects; 7 . keep up-to-date with organizational policies and norms

Editability: 8. edit others' information after they have posted it; 9. edit my information after I have posted it; 10 . create or edit a document collaboratively Persistence: 11. find information about prior NPB projects; 12 . maintain relations with others at NPB despite changes in activities, work, or location; 13. have my information or comments stay available after I post them

Personalization: 14. include the information, photos, and other content on NPB media that present my personal identity; 15 . adjust my NPB media profile to my preferences Pervasiveness: 16. get responses to my requests from others quickly; 17 . communicate with others while moving, commuting, traveling; 18. communicate with infrequent or less important work relationships

Searchability: 19. search for information or people by entering search words; 20. search for information or people by following links between contents; 21. search for tags or keywords that someone else has added to content

Sharing: 22. create groups for sharing information about specific projects, departments, or teams; 23. obtain and use others' files, documents, photos, other information; 24. share my files, documents, photos, other information with other NPB employees Evaluatability: 25. evaluate other people's information by providing my recommendations, comments, liking, or tagging; 26. see other people's evaluation of information through their recommendations, comments, liking, or tagging Visibility: 27. see other people's answers to other people's questions; 28. see who has interactions or links with particular employees or their information; 29. see the number of others who have "liked" or linked to the same content

Signaling: 30. receive notifications about other information or updates that are similar to what I have just been looking at; 31. receive notifications about other people's information or updates

Note: "Think about the extent to which you agree that these activities are currently possible (whether you actually do them or not), using the various media (email, phones, instant messaging, intranet, social media, etc.) available at NPB. Throughout, 'others' and 'people' refer to current employees of NPB." Then, "To what extent do you agree with the following statements? It is currently possible for me to..." Response choices for each item were 1 Strongly disagree, through 4 neither agree or disagree, to 7 strongly agree, and 8 do not know (recoded as missing). The tentative affordance labels here were used only for initial grouping; they were not included on the survey. 
Table 2

Media Affordances Descriptives, Components, and Loadings

\begin{tabular}{|c|c|c|c|c|c|c|c|c|}
\hline Item & $\mathbf{N}$ & $\mathbf{M}$ & SD & $\begin{array}{c}\text { Visibili } \\
\text { ty }\end{array}$ & $\begin{array}{c}\text { Editab } \\
\text { ility; } \\
\text { Self- } \\
\text { Presen } \\
\text { tation }^{\text {a }} \\
\end{array}$ & $\begin{array}{c}\text { Aware } \\
\text { ness }\end{array}$ & $\begin{array}{l}\text { Pervas } \\
\text { iveness }\end{array}$ & $\begin{array}{l}\text { Search } \\
\text { ability }\end{array}$ \\
\hline links info new info & 435 & 5.3 & 1.40 & .19 & .22 & .46 & .58 & .03 \\
\hline links people new people & 435 & 5.1 & 1.38 & .19 & .33 & .43 & .53 & .06 \\
\hline info others in dept & 435 & 4.9 & 1.44 & .11 & .23 & .74 & .34 & .08 \\
\hline info others outside dept & 428 & 4.5 & 1.45 & .23 & .13 & .75 & .30 & .12 \\
\hline activities, opinion, locations of others & 436 & 4.6 & 1.39 & .32 & .22 & .69 & .16 & -.01 \\
\hline keep up-to-date progress of projects & 433 & 4.5 & 1.48 & .17 & .13 & .78 & .08 & .26 \\
\hline keep up-to-date org policies \& norms & 436 & 4.5 & 1.50 & .25 & .19 & .64 & .02 & .33 \\
\hline edit others' info after they post & 420 & 4.7 & 1.44 & .18 & {$[.59$} & .30 & .10 & .26 \\
\hline edit my info after I post & 418 & 5.2 & 1.32 & .22 & .71 & .20 & .16 & .15 \\
\hline edit document collaboratively & 415 & 5.8 & 1.26 & .21 & $.75]$ & .17 & .33 & .00 \\
\hline find info prior NPB projects & 423 & 4.3 & 1.52 & .16 & .29 & .52 & -.05 & .55 \\
\hline maintain relations w/others at org & 424 & 5.2 & 1.29 & .17 & {$[.56$} & .39 & .13 & .32 \\
\hline my info comments stay available & 417 & 5.3 & 1.34 & .23 & .67 & .33 & .14 & .23 \\
\hline include info present my identity & 419 & 5.0 & 1.51 & .33 & 69 & .18 & .26 & .11 \\
\hline adjust media profile my preferences & 416 & 5.1 & 1.48 & .27 & $.72]$ & .18 & .27 & .11 \\
\hline get responses quickly & 448 & 5.6 & 1.17 & .15 & .26 & .17 & .65 & .33 \\
\hline comm while moving & 442 & 5.9 & 1.08 & .21 & .39 & .09 & .70 & .21 \\
\hline comm infrequent work relations & 428 & 5.5 & 1.20 & .18 & .27 & .22 & .76 & .20 \\
\hline search words & 433 & 5.2 & 1.48 & .24 & .20 & .27 & .42 & .67 \\
\hline follow links & 410 & 5.0 & 1.46 & .26 & .29 & .27 & .30 & .71 \\
\hline tags keywords others have added & 407 & 4.6 & 1.50 & .48 & .15 & .14 & .33 & .66 \\
\hline create groups & 416 & 5.3 & 1.37 & .48 & .50 & .03 & .34 & .30 \\
\hline obtain others' info & 416 & 5.0 & 1.46 & .44 & .42 & .11 & .22 & .43 \\
\hline my info with other employees & 426 & 5.6 & 1.30 & .44 & .63 & -.07 & .38 & .15 \\
\hline $\begin{array}{l}\text { provide my recommendations \& } \\
\text { comments }\end{array}$ & 394 & 4.8 & 1.52 & .78 & .29 & .18 & .14 & .26 \\
\hline see others' evaluations & 393 & 4.6 & 1.47 & .83 & .23 & .22 & .14 & .24 \\
\hline see others' answers to others' questions & 406 & 5.0 & 1.37 & .72 & .31 & .16 & .23 & .17 \\
\hline see interactions among employees & 372 & 4.3 & 1.48 & .77 & .18 & .31 & .10 & .18 \\
\hline see number others liked or linked & 379 & 4.5 & 1.54 & .84 & .24 & .23 & .13 & .05 \\
\hline receive notifications about similar info & 362 & 4.3 & 1.48 & .85 & .14 & .22 & .09 & .18 \\
\hline receive notifications about others' info & 390 & 4.6 & 1.50 & .85 & .27 & .14 & .18 & .04 \\
\hline \multicolumn{4}{|c|}{ Eigenvalue } & 6.4 & 5.3 & 4.3 & 3.5 & 2.9 \\
\hline \multicolumn{4}{|c|}{ Variance explained } & $20.5 \%$ & 16.9 & 14.0 & 11.3 & 9.2 \\
\hline \multicolumn{4}{|c|}{$\alpha$} & .96 & $\begin{array}{c}.89 \\
{[.83,} \\
.89]\end{array}$ & .87 & .84 & .89 \\
\hline \multicolumn{4}{|c|}{ M } & 4.70 & 5.21 & 4.60 & 5.70 & 4.94 \\
\hline
\end{tabular}




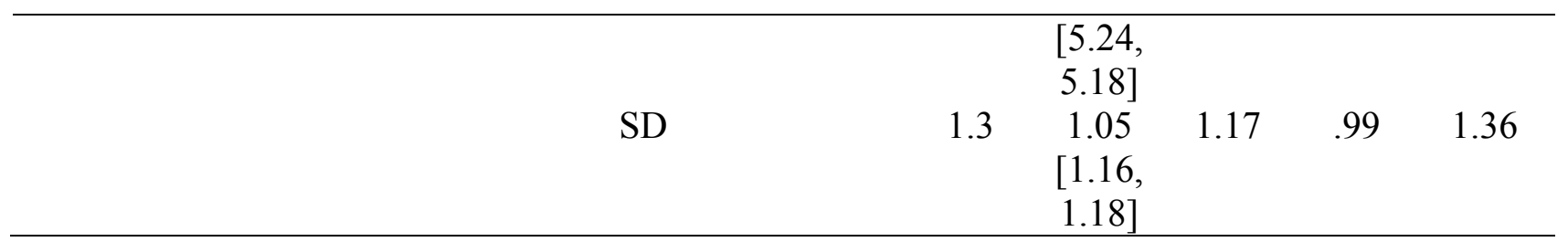

Note:

Principal components analysis, varimax rotation.

The loadings and scale descriptives are based on the exploratory principal components analysis results. As noted in the text, based on the confirmatory factor analysis measurement model, four items were removed, and the revised mean scales were used in the analyses.

a. The second component included loadings that correspond to the distinct concepts of editability and self-presentation, so the table shows the means, SDs, and alpha reliabilities for the full component scale as well as the two separate scales. Analyses use these two separate affordances.

Table 3

Reliability and Convergent and Discriminant Validity of Affordance Constructs

\begin{tabular}{|c|c|c|c|c|c|c|c|c|c|}
\hline Affordance & $\boldsymbol{\alpha}$ & CR & AVE & $\begin{array}{c}\text { Visibil } \\
\text { ity }\end{array}$ & $\begin{array}{l}\text { Edita } \\
\text { bility }\end{array}$ & $\begin{array}{c}\text { Self- } \\
\text { present } \\
\text { ation }\end{array}$ & $\begin{array}{c}\text { Aware } \\
\text { ness }\end{array}$ & $\begin{array}{c}\text { Pervasive } \\
\text { ness }\end{array}$ & $\begin{array}{c}\text { Search } \\
\text { ability }\end{array}$ \\
\hline Visibility & .96 & .95 & .77 & .88 & -- & -- & -- & -- & -- \\
\hline Editability & .83 & .84 & .63 & .48 & .79 & -- & -- & -- & -- \\
\hline $\begin{array}{l}\text { Self- } \\
\text { presentation }\end{array}$ & .89 & .85 & .66 & .57 & .65 & .81 & -- & -- & -- \\
\hline Awareness & .87 & .85 & .65 & .34 & .44 & .44 & .81 & -- & -- \\
\hline Pervasiveness & .84 & .82 & .60 & .56 & .55 & .60 & .37 & .77 & -- \\
\hline Searchability & .89 & .85 & .70 & .66 & .49 & .53 & .53 & .66 & .84 \\
\hline
\end{tabular}

Note: $\alpha$ : Cronbach alpha; CR: composite reliability; AVE: average variance extracted;

Diagonals: square root of AVE; Off-diagonals: correlations 
Table 4

Organizational Media Use Descriptives, Components, and Loadings

\begin{tabular}{|c|c|c|c|c|c|c|c|c|c|c|c|c|}
\hline Item & $\mathbf{N}$ & $\mathbf{M}$ & SD & Conf & $\begin{array}{c}\text { Basic } \\
\text { Out }\end{array}$ & $\begin{array}{c}\text { Ext } \\
\text { Soc } \\
\text { Med }\end{array}$ & $\begin{array}{c}\text { Basic } \\
\text { Sup }\end{array}$ & $\begin{array}{c}\text { Whats } \\
\text { App }\end{array}$ & $\begin{array}{c}\text { Intra } \\
\text { net }\end{array}$ & $\begin{array}{c}\text { Basic } \\
\text { In } 1 \\
\end{array}$ & $\begin{array}{c}\text { Basic } \\
\text { In } 2 \\
\end{array}$ & $\begin{array}{c}\text { Text } \\
\text { ing }\end{array}$ \\
\hline \multicolumn{13}{|l|}{ Within Organization } \\
\hline Face-to-face & 460 & 6.5 & 2.28 & .07 & .79 & .11 & -.05 & -.03 & .06 & .00 & .04 & -.16 \\
\hline FtF Meeting & 455 & 4.5 & 2.07 & .29 & .72 & .10 & .10 & .05 & .01 & .26 & -.02 & -.04 \\
\hline Email & 461 & 7.6 & 1.91 & .07 & .81 & .01 & .10 & .11 & .12 & .07 & .14 & .07 \\
\hline Phone & 460 & 6.3 & 2.03 & .02 & .79 & -.03 & .14 & .08 & .07 & .02 & .33 & .09 \\
\hline Short text & 461 & 5.2 & 2.49 & .18 & .60 & .17 & .04 & .19 & .07 & .07 & .02 & .57 \\
\hline Conferencing no video & 460 & 2.5 & 1.70 & .59 & .44 & -.05 & .07 & .15 & .01 & .12 & -.01 & .19 \\
\hline Conferencing video & 461 & 2.5 & 1.65 & .65 & .46 & .01 & .12 & .07 & .05 & .12 & -.16 & .15 \\
\hline Intranet & 458 & 2.3 & 1.86 & .07 & .31 & .03 & -.01 & .10 & .70 & -.08 & -.03 & .16 \\
\hline WhatsApp & 453 & 2.5 & 2.39 & .01 & .23 & .13 & -.04 & .84 & .08 & -.02 & .02 & .05 \\
\hline External social media & 460 & 4.0 & 2.71 & .09 & .21 & .79 & -.02 & .21 & .12 & .03 & -.06 & .15 \\
\hline \multicolumn{13}{|c|}{ Within Department } \\
\hline Face-to-face & 458 & 8.4 & 1.29 & -.10 & .13 & -.09 & .09 & .02 & .04 & .74 & .10 & .09 \\
\hline FtF Meeting & 457 & 5.7 & 1.76 & .24 & .10 & .21 & .20 & .04 & -.01 & .76 & .09 & .08 \\
\hline Email & 457 & 7.8 & 1.50 & .14 & .10 & .08 & .11 & .09 & .18 & .41 & .50 & .16 \\
\hline Phone & 457 & 6.3 & 1.91 & .11 & .19 & .02 & .03 & .02 & .05 & .14 & .86 & .06 \\
\hline Short text & 457 & 5.6 & 2.69 & .25 & -.03 & .21 & -.03 & .01 & .04 & .23 & .19 & .80 \\
\hline Conferencing no video & 453 & 2.4 & 1.85 & .78 & .10 & .03 & -.08 & .09 & .10 & .14 & .17 & .10 \\
\hline Conferencing video & 455 & 2.4 & 1.75 & .81 & .10 & .08 & .01 & .01 & .05 & .12 & .06 & .06 \\
\hline Intranet & 457 & 1.7 & 1.39 & .12 & .02 & .03 & .00 & -.01 & .88 & .07 & .11 & -.02 \\
\hline WhatsApp & 456 & 2.3 & 2.21 & .04 & .02 & .24 & -.03 & .84 & -.05 & .09 & .11 & .02 \\
\hline External social media & 456 & 3.5 & 2.72 & .05 & .02 & .89 & -.02 & .18 & .04 & .09 & .10 & .09 \\
\hline \multicolumn{13}{|c|}{ With Supervisor } \\
\hline Face-to-face & 458 & 6.0 & 2.03 & -.09 & .06 & -.03 & .82 & -.01 & .07 & .29 & -.08 & .03 \\
\hline FtF Meeting & 457 & 4.8 & 1.55 & .17 & .05 & .07 & .60 & -.02 & -.06 & .57 & -.02 & .03 \\
\hline Email & 456 & 6.1 & 1.61 & .09 & .14 & .13 & .77 & .10 & .09 & .08 & .32 & .14 \\
\hline Phone & 455 & 4.5 & 1.87 & .09 & .18 & .14 & .56 & .10 & -.03 & -.12 & .59 & .09 \\
\hline Short text & 455 & 3.7 & 2.32 & .28 & -.04 & .21 & .42 & .09 & .03 & .02 & .03 & .71 \\
\hline Conferencing no video & 456 & 1.8 & 1.39 & .79 & .00 & .09 & .04 & .10 & .16 & -.10 & .10 & .13 \\
\hline Conferencing video & 457 & 1.8 & 1.44 & .81 & .03 & .12 & .08 & -.03 & .14 & -.06 & .04 & .05 \\
\hline Intranet & 457 & 1.3 & 0.87 & .22 & -.01 & .13 & .09 & -.06 & .75 & .05 & .05 & -.04 \\
\hline WhatsApp & 455 & 1.5 & 1.57 & .18 & .01 & .17 & .18 & .78 & -.02 & .02 & -.01 & .06 \\
\hline External social media & 457 & 2.2 & 1.93 & .12 & -.01 & .76 & .24 & .21 & .06 & .00 & .08 & .13 \\
\hline \multicolumn{3}{|l|}{ Eigenvalue } & & 3.9 & 3.5 & 2.4 & 2.3 & 2.3 & 2.0 & 2.0 & 1.8 & 1.7 \\
\hline \multicolumn{3}{|l|}{$\%$ Variance explained } & & 12.8 & 11.8 & 7.9 & 7.8 & 7.7 & 6.7 & 6.5 & 5.9 & 5.8 \\
\hline \multicolumn{3}{|c|}{$\alpha$} & & .88 & .84 & .83 & .77 & .81 & .69 & .57 & .60 & .75 \\
\hline \multicolumn{3}{|c|}{$\mathrm{M}$} & & 2.25 & 6.22 & 3.23 & 5.34 & 1.77 & 2.07 & 7.04 & 7.04 & 4.85 \\
\hline \multicolumn{3}{|c|}{ SD } & & 1.30 & 1.71 & 2.15 & 1.38 & 1.12 & 1.77 & 1.29 & 1.45 & 2.04 \\
\hline
\end{tabular}




\begin{tabular}{rc}
\hline Combining Basic & \\
within Department 1 & Basic \\
and 2 & In \\
$\alpha$ & .64 \\
$\mathrm{M}$ & 7.04 \\
$\mathrm{SD}$ & 1.13 \\
\hline
\end{tabular}

Note: Principal components analysis, varimax rotation

Scale: 1 Never, 2 a few times a year or less, 3 once a month or less, 4 a few times a month, 5 once a week, 6 a few times a week, 7 every day, 8 a few times a day, 9 many times a day.

Media and usage measures adapted from Boswell and Olson-Buchanan (2007) and specified through discussions with the organizational contact.

Table 5

Correlations between Organizational Media Use and Affordances

\begin{tabular}{lcccccc}
\cline { 2 - 7 } Media Use & \multicolumn{7}{c}{ Affordances } \\
\hline Conferencing & Visibility & $\begin{array}{c}\text { Edit } \\
\text { ability }\end{array}$ & $\begin{array}{c}\text { Self- } \\
\text { presentation }\end{array}$ & $\begin{array}{c}\text { Aware } \\
\text { ness }\end{array}$ & $\begin{array}{c}\text { Pervasive } \\
\text { ness }\end{array}$ & $\begin{array}{c}\text { Search } \\
\text { ability }\end{array}$ \\
Basic organizational & $.11^{* *}$ & $.12^{*}$ & $.17^{* * *}$ & $.19^{* * *}$ & $.17^{*}$ & .08 \\
External social media & .03 & $.11^{*}$ & .08 & $.11^{*}$ & $.12^{*}$ & -.00 \\
Basic supervisor & $.12^{* *}$ & .09 & $.12^{* *}$ & $.17^{* * *}$ & $.12^{* *}$ & $.10^{*}$ \\
Intranet & -.06 & .04 & .00 & $.14^{* * *}$ & .07 & .02 \\
WhatsApp & $.10^{*}$ & .04 & .08 & $.14^{* * *}$ & .03 & $.11^{*}$ \\
Basic department & .07 & -.02 & .05 & .07 & $.12^{*}$ & .01 \\
Texting & .02 & $.09 *$ & .07 & $.10^{*}$ & $.17^{* * *}$ & .08 \\
\hline
\end{tabular}

$\mathrm{N}=429-449$

Pearson correlations; two-tailed significance tests

$* p<.05 ; * * p<.01 ; * * * p<.001$ 


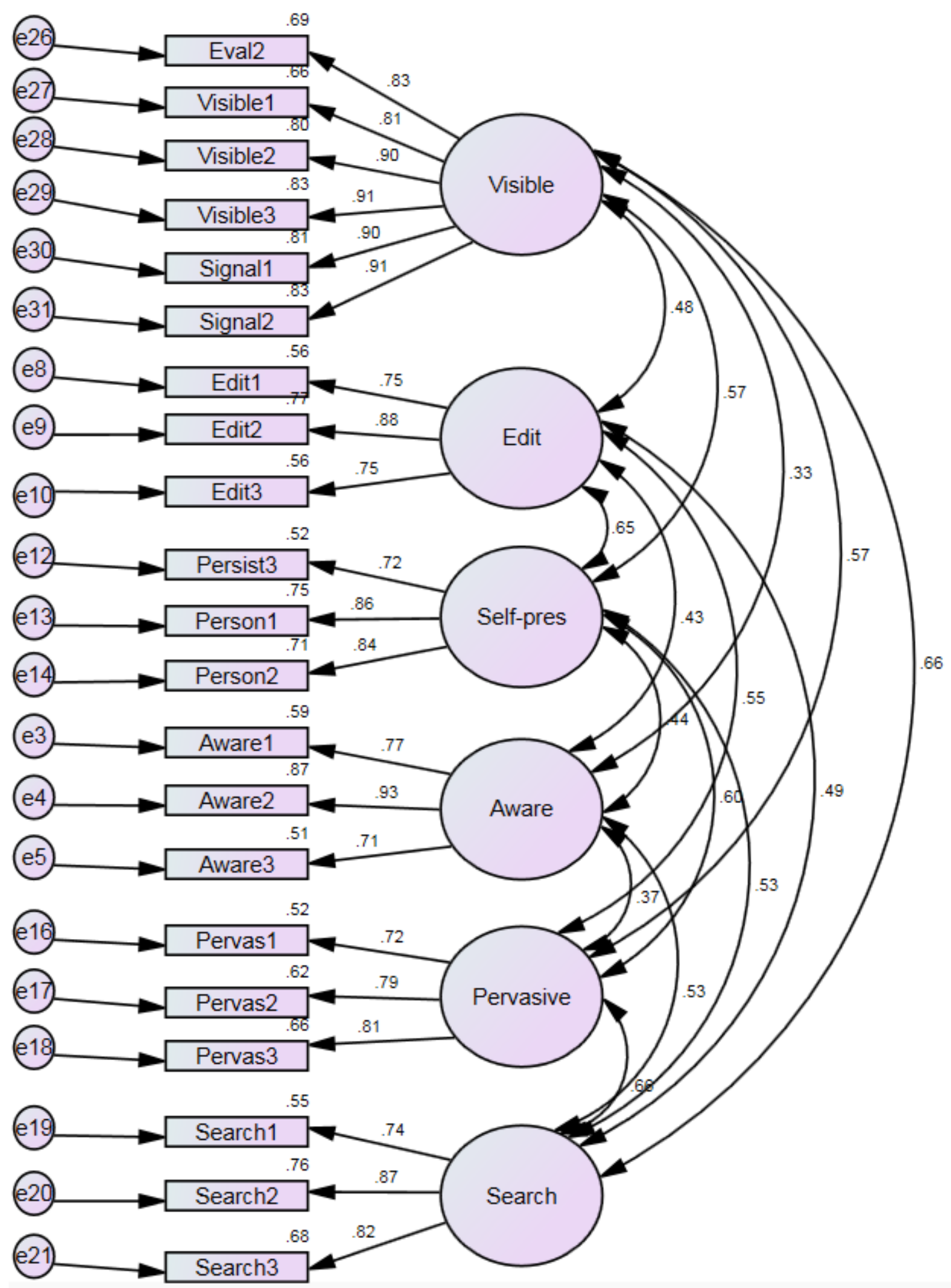

Figure 1. Revised affordances measurement model. 